\title{
Centrality Dependence of Directed and Elliptic Flow at the SPS
}

\author{
A.M. Poskanzer ${ }^{\mathrm{a}}$ and S.A. Voloshin ${ }^{\mathrm{a}}$ for the NA49 Collaboration
}

${ }^{a}$ Lawrence Berkeley National Laboratory, Berkeley, CA 94720, USA

J. Bächler ${ }^{6}$, D. Barna ${ }^{5}$, L.S. Barnby ${ }^{3}$, J. Bartke ${ }^{7}$, R.A. Barton ${ }^{3}$, L. Betev ${ }^{14}$, H. Białkowska $^{17}$, A. Billmeier ${ }^{11}$, C. Blume ${ }^{8}$, C.O. Blyth ${ }^{3}$, B. Boimska ${ }^{17}$, J. Bracinik ${ }^{4}$, F.P. Brady ${ }^{9}$, R. Brockmann ${ }^{8, \dagger}$, R. Brun ${ }^{6}$, P. Bunčić ${ }^{6,11}$, L. Carr ${ }^{19}$, D. Cebra ${ }^{9}$, G.E. Cooper ${ }^{2}$, J.G. Cramer ${ }^{19}$, P. Csató ${ }^{5}$, V. Eckardt ${ }^{16}$, F. Eckhardt ${ }^{15}$, D. Ferenc ${ }^{9}$, H.G. Fischer ${ }^{6}$, Z. Fodor ${ }^{5}$, P. Foka ${ }^{11}$, P. Freund ${ }^{16}$, V. Friese ${ }^{15}$, J. Ftacnik ${ }^{4}$, J. Gál ${ }^{5}$, R. Ganz ${ }^{16}$, M. Gaździcki ${ }^{11}$, E. Gładysz ${ }^{7}$, J. Grebieszkow ${ }^{18}$, J.W. Harris ${ }^{20}$, S. Hegyi ${ }^{5}$, V. Hlinka ${ }^{4}$, C. Höhne ${ }^{15}$, G. Igo ${ }^{14}$, M. Ivanov ${ }^{4}$, P. Jacobs², R. Janik ${ }^{4}$, P.G. Jones ${ }^{3}$, K. Kadija ${ }^{21,16}$, V.I. Kolesnikov ${ }^{10}$, M. Kowalski ${ }^{7}$, B. Lasiuk $^{20}$, P. Lévai ${ }^{5}$, A.I. Malakhov ${ }^{10}$, S. Margetis ${ }^{13}$, C. Markert ${ }^{8}$, B.W. Mayes ${ }^{12}$, G.L. Melkumov ${ }^{10}$, J. Molnár ${ }^{5}$, J.M. Nelson ${ }^{3}$, G. Odyniec ${ }^{2}$, M.D. Oldenburg ${ }^{11}$, G. Pálla ${ }^{5}$, A.D. Panagiotou ${ }^{1}$, A. Petridis ${ }^{1}$, M. Pikna ${ }^{4}$, L. Pinsky ${ }^{12}$, A.M. Poskanzer ${ }^{2}$, D.J. Prindle ${ }^{19}$, F. Pühlhofer ${ }^{15}$, J.G. Reid ${ }^{19}$, R. Renfordt ${ }^{11}$, W. Retyk ${ }^{18}$, H.G. Ritter ${ }^{2}$, D. Röhrich ${ }^{11, *}$, C. Roland $^{8}$, G. Roland ${ }^{11}$, A. Rybicki ${ }^{7}$, T. Sammer ${ }^{16}$, A. Sandoval ${ }^{8}$, H. Sann ${ }^{8}$, A.Yu. Semenov ${ }^{10}$, E. Schäfer ${ }^{16}$, N. Schmitz ${ }^{16}$, P. Seyboth ${ }^{16}$, F. Siklér ${ }^{5,6}$, B. Sitar ${ }^{4}$, E. Skrzypczak ${ }^{18}$, R. Snellings ${ }^{2}$, G.T.A. Squier ${ }^{3}$, R. Stock ${ }^{11}$, P. Strmen ${ }^{4}$, H. Ströbele ${ }^{11}$, T. Susa ${ }^{21}$, I. Szarka ${ }^{4}$, I. Szentpétery ${ }^{5}$,

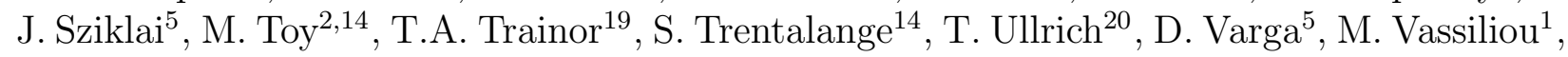

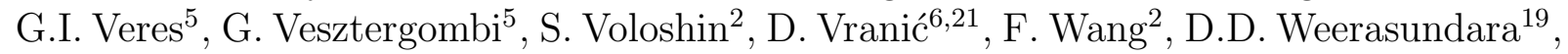
S. Wenig ${ }^{6}$, C. Whitten ${ }^{14}$, N. Xu ${ }^{2}$, T.A. Yates ${ }^{3}$, I.K. Yoo ${ }^{15}$, J. Zimányi ${ }^{5}$

${ }^{1}$ Department of Physics, University of Athens, Athens, Greece.

${ }^{2}$ Lawrence Berkeley National Laboratory, University of California, Berkeley, USA.

${ }^{3}$ Birmingham University, Birmingham, England.

${ }^{4}$ Institute of Physics, Bratislava, Slovakia.

${ }^{5}$ KFKI Research Institute for Particle and Nuclear Physics, Budapest, Hungary.

${ }^{6}$ CERN, Geneva, Switzerland.

${ }^{7}$ Institute of Nuclear Physics, Cracow, Poland.

${ }^{8}$ Gesellschaft für Schwerionenforschung (GSI), Darmstadt, Germany.

${ }^{9}$ University of California at Davis, Davis, USA.

${ }^{10}$ Joint Institute for Nuclear Research, Dubna, Russia.

${ }^{11}$ Fachbereich Physik der Universität, Frankfurt, Germany.

${ }^{12}$ University of Houston, Houston, TX, USA.

${ }^{13}$ Kent State University, Kent, OH, USA.

${ }^{14}$ University of California at Los Angeles, Los Angeles, USA.

${ }^{15}$ Fachbereich Physik der Universität, Marburg, Germany.

${ }^{16}$ Max-Planck-Institut für Physik, Munich, Germany.

${ }^{17}$ Institute for Nuclear Studies, Warsaw, Poland. 
${ }^{18}$ Institute for Experimental Physics, University of Warsaw, Warsaw, Poland.

${ }^{19}$ Nuclear Physics Laboratory, University of Washington, Seattle, WA, USA.

${ }^{20}$ Yale University, New Haven, CT, USA.

${ }^{21}$ Rudjer Boskovic Institute, Zagreb, Croatia.

*Present address: University of Bergen, Norway.

${ }^{\dagger}$ deceased.

New data with a minimum bias trigger for $158 \mathrm{GeV} /$ nucleon $\mathrm{Pb}+\mathrm{Pb}$ have been analyzed. Directed and elliptic flow as a function of rapidity of the particles and centrality of the collision are presented. The centrality dependence of the ratio of elliptic flow to the initial space elliptic anisotropy is compared to models.

\section{Motivation}

In the Fourier decomposition of the azimuthal distribution of particles, the first and second coefficients correspond to the directed, $v_{1}$, and elliptic, $v_{2}$, flow, respectively [1]. The elliptic flow is expected to be sensitive to the system evolution at the time of maximum compression [2]. Ollitrault [3] showed that in a hydro model the elliptic flow is proportional to the initial space elliptic anisotropy of the overlapping region weighted by the number of nucleon collisions in the beam direction. This initial space elliptic anisotropy, which we will call $\varepsilon$, has been calculated for a Woods-Saxon density distribution and shown to be almost insensitive to the nucleon-nucleon cross section [4]. It is enlightening to plot $v_{2} / \varepsilon$ versus centrality [5,6] in order to look for changes in the reaction mechanism or properties of the nuclear matter. Thus the motivation for this work is to find a signature (elliptic flow), scan this signature as a function of a control parameter (centrality), and, after first dividing out the geometry of the initial state, look for a change in the physics (unexpected behavior).

\section{Experiment}

NA49 has published directed and elliptic flow results from the NA49 Main Time Projection Chambers for a set of data taken with a medium impact parameter trigger $[7,8]$. We now have a new set of data taken with a minimum bias trigger so that we can study the flow centrality dependence. Also, the tracks from the Main and Vertex TPCs are combined resulting in full coverage of the forward hemisphere. The data in the graphs below presenting flow as a function of rapidity have been reflected about mid-rapidity. The data have been integrated over $p_{t}$ and in some cases also over $y$ using as weights the measured double differential cross sections [9,10]. The data have been sorted into six centrality bins using the Zero Degree Calorimeter, with "cen1" being the most central and "cen6" the most peripheral. The impact parameter values for these bins have been estimated from the number of participants which were obtained by integrating the yields [9.10]. Slightly higher values of $b$, used in the oral presentation of this paper, were determined from the fraction of the total cross section corresponding to each bin. Only some of the available data has been analyzed so far. Thus these data are preliminary and no systematic errors have been included yet. 


\section{Results}

The rapidity dependence of directed and elliptic flow integrated over the whole range of measured impact parameters up to about $11 \mathrm{fm}$ is shown in Fig. 11. The pion $v_{1}$ values hug the axis near mid-rapidity and the $v_{2}$ values for both pions and protons appear to slightly peak somewhat away from mid-rapidity. For pions the $v_{1}$ and $v_{2}$ values are shown for different centrality bins in Fig. 2. Both sets of flow values increase continuously as the reaction becomes more peripheral. The elliptic flow values for pions have been integrated over rapidity up to $y=6$ and are shown in Fig. 3, together with simulations from RQMD v2.3 [1]. The flow from RQMD peaks at a medium impact parameter whereas the flow from experiment continues to rise. In Fig. 囵 the $v_{2}$ values have been divided by the initial space elliptic anisotropy [4. In addition, results from RQMD v3.0 [5] which includes a phase transition are shown. Typical hydro results [12] are also shown. The data are below hydro indicating a lack of complete equilibration in the reaction [13]. The data are above the RQMD resonance gas and tantalizingly close to the RQMD phase transition calculation. Clearly, it is important to process the full set of NA49 data and obtain final results.
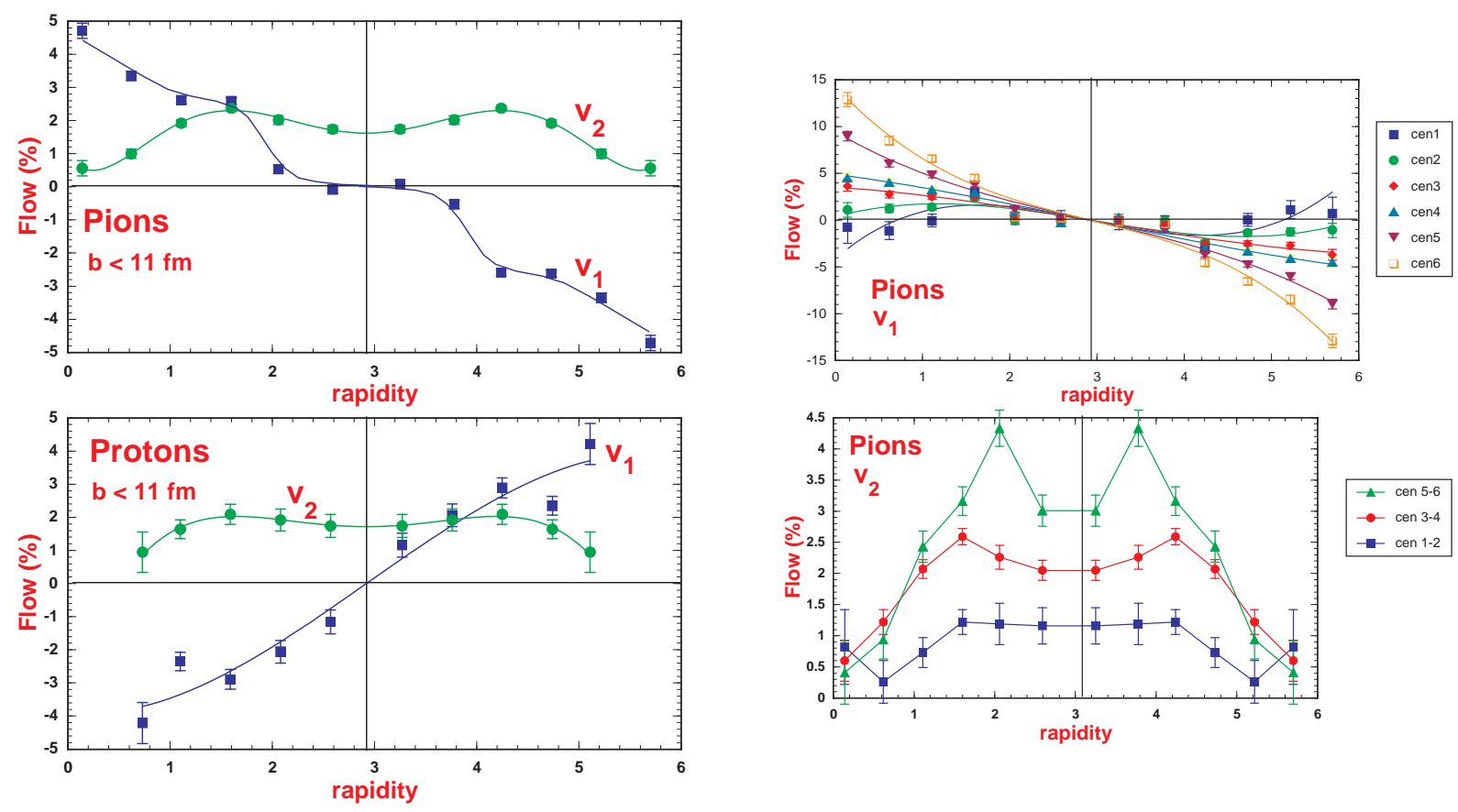

Figure 2. Pion directed and elliptic flow

Figure 1. Pion and proton directed and elliptic flow versus rapidity.

for different centralities where "cen1" is the most central and "cen6" the most peripheral. 


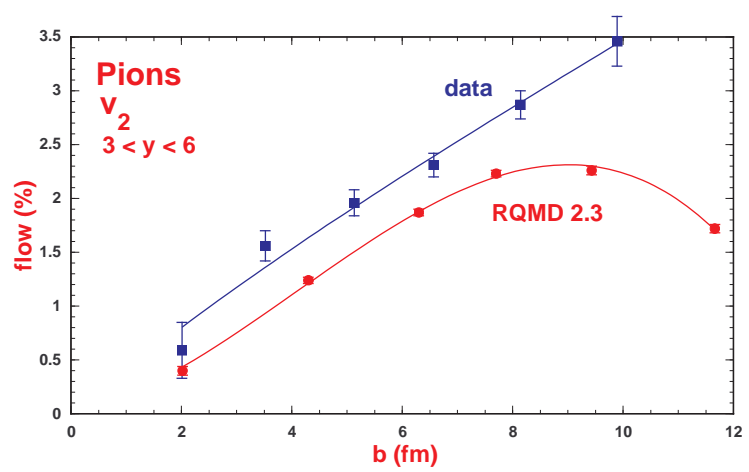

Figure 3. Pion elliptic flow versus the impact parameter.

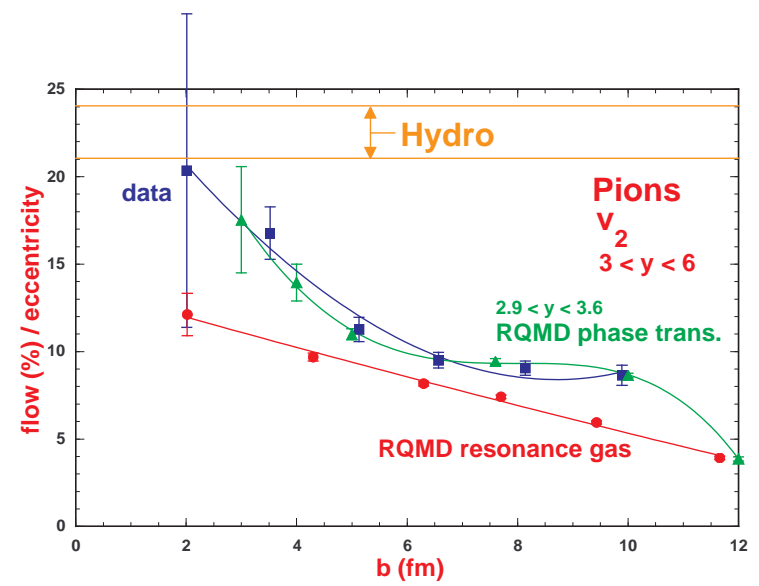

Figure 4. Pion elliptic flow divided by the initial space elliptic anisotropy, $v_{2} / \varepsilon$, versus the impact parameter.

\section{REFERENCES}

1. A.M. Poskanzer and S.A. Voloshin, Phys. Rev. C 58, 1671 (1998).

2. H. Sorge, Phys. Rev. Letters 78, 2309 (1997).

3. J.-Y. Ollitrault, Phys. Rev. D 46, 229 (1992).

4. P. Jacobs and G. Cooper, STAR Note SN0402 (1999).

5. H. Sorge, Phys. Rev. Lett. 82, 2048 (1999); H. Sorge, this conference (1999).

6. H. Heiselberg and A.-M. Levy, Phys. Rev. C 59, 2716 (1999).

7. H. Appelshäuser et al., NA49, Phys. Rev. Letters 80, 4136 (1998).

8. A.M. Poskanzer et al., NA49, Nucl. Phys. A638, 463c (1998).

9. G. Cooper, NA49, this conference.

10. F. Sikler, NA49, this conference.

11. H. Sorge, Phys. Rev. C 52, 3291 (1995).

12. P. Kolb, J. Sollfrank, U. Heinz, preprint nucl-th/9906003.

13. S.A. Voloshin and A.M. Poskanzer, preprint nucl-th/9906075. 